\title{
Software development for estimation of optical clearing agent's diffusion coefficients in biological tissues
}

\author{
Pedro Peixoto ${ }^{1}$, Luís Oliveira ${ }^{1,2,3^{*}}$, Maria Inês Carvalho ${ }^{2,4}$, Elisabete Nogueira ${ }^{1,3}$, \\ Valery V. Tuchin ${ }^{5,6,7}$ \\ ${ }^{1}$ Physics Department - Polytechnic Institute of Porto, School of Engineering, Rua Dr. António Bernardino de Almeida, \\ 431, 4200-072 Porto, Portugal \\ 2 FEUP - University of Porto, Rua Dr. Roberto Frias, 4200-465 Porto, Portugal \\ ${ }^{3} \mathrm{CIETI}$ - Centre of Innovation in Engineering and Industrial Technology, ISEP, Rua Dr. António Bernardino de Almeida, \\ 431, 4200-072 Porto, Portugal \\ ${ }^{4}$ DEEC/FEUP and INESC TEC, University of Porto, Rua Dr. Roberto Frias, 4200-465 Porto, Portugal \\ ${ }^{5}$ Research-Educational Institute of Optics and Biophotonics, Saratov National Research State University, \\ 83 Astrakhanskaya str., Saratov 410012, Russia \\ ${ }^{6}$ Laboratory of Laser Diagnostics of Technical and Living Systems, Institute of Precise Mechanics and Control RAS, \\ 24 Rabochaya str., Saratov 410028, Russia \\ 7 Interdisciplinary Laboratory of Biophotonics, Tomsk National Research State University, 36 Lenin's av., \\ Tomsk 634050, Russia \\ *e-mail: Imo@isep.ipp.pt
}

Abstract. The study of chemical diffusion in biological tissues is a research field of high importance and with application in many clinical, research and industrial areas. The evaluation of diffusion and viscosity properties of chemicals in tissues is necessary to characterize treatments or inclusion of preservatives in tissues or organs for low temperature conservation. Recently, we have demonstrated experimentally that the diffusion properties and dynamic viscosity of sugars and alcohols can be evaluated from optical measurements. Our studies were performed in skeletal muscle, but our results have revealed that the same methodology can be used with other tissues and different chemicals. Considering the significant number of studies that can be made with this method, it becomes necessary to turn data processing and calculation easier. With this objective, we have developed a software application that integrates all processing and calculations, turning the researcher work easier and faster. Using the same experimental data that previously was used to estimate the diffusion and viscosity of glucose in skeletal muscle, we have repeated the calculations with the new application. Comparing between the results obtained with the new application and with previous independent routines we have demonstrated great similarity and consequently validated the application. This new tool is now available to be used in similar research to obtain the diffusion properties of other chemicals in different tissues or organs. (C) 2016 Samara State Aerospace University (SSAU).

Keywords: collimated optical transmittance, chemical diffusion in tissues, glucose, optical clearing, refractive index matching, thickness variation, viscosity, software application.

Paper \#2815 received 2015.12.17; revised manuscript received 2015.12.30; accepted for publication 2015.12.31; published online 2016.02.05. 


\section{References}

1. F. E. Dunlap, O. H. Mills, M. R. Tuley, M. D. Baker, and R. T. Plott, "Adapalene $0.1 \%$ gel for the treatment of acne vulgaris: its superiority compared to tretinoin $0.025 \%$ cream in skin tolerance and patient preference," British Journal of Dermatology 139(52), 17-22 (1998).

2. E. A. Genina, A. N. Bashkatov, Y. P. Sinichkin, I. Y. Yanina, and V. V. Tuchin, "Optical clearing of biological tissues: prospects of application in medical diagnostics and phototherapy," J. of Biomedical Photonics and Engineering 1(1), 22-58 (2015).

3. L. M. Alvarez, Compendium of Organ \& Tissue Banking Concepts (2015).

4. R. Bertram, and M. Pernarowski, "Glucose diffusion in pancreatic islets of Langerhans," Biophys. J. 74(4), 1722-1731 (1998).

5. A. C. Ribeiro, O. Ortona, S. M. N. Simões, C. I. A. V. Santos, P. M. R. A. Prazeres, A. J. M. Valente, V. M. M. Lobo, and H. D. Burrows, "Binary mutual diffusion coefficients of aqueous solutions of sucrose, lactose, glucose and fructose in the temperature range from (298.15 to 328.15) K," J. Chem. Eng. Data 51(5), 18361840 (2006).

6. M. G. Ghosn, E. F. Carbajal, N. A. Befrui, V. V. Tuchin, and K. V. Larin, "Concentration effect on the diffusion of glucose in ocular tissues," Opt. Laser Eng. 46(12), 911-914, (2008).

7. L. M. Oliveira, M. I. Carvalho, E. M. Nogueira, and V. V. Tuchin, "The characteristic time of glucose diffusion measured for muscle tissue at optical clearing," Laser Physics 23(7), 075606 (2013).

8. A. N. Bashkatov, E. A. Genina, and V. V. Tuchin, "Measurement of glucose diffusion coefficients in human tissues," Chap. 19 in Handbook of Optical Sensing of Glucose in Biological Fluids and Tissues, V. V. Tuchin, (ed.), Taylor \& Francis Group LLC, CRC Press, London, 587-621 (2009).

9. M. Kreft, M. Lukšič, T. M. Zorec, M. Prebil, and R. Zorec, "Diffusion of D-glucose measured in the cytosol of a single astrocyte," Cell. Mol. Life Sci. 70(8), 1483-1492 (2013).

10. L. Oliveira et al., "Optical clearing mechanisms characterization in muscle", to be published.

11. V. V. Tuchin, I. L. Maksimova, D. A. Zimnyakov, I. L. Kon, A. H. Mavlyutov, and A. A. Mishin, "Light propagation in tissues with controlled optical properties," J. Biomed. Opt. 2(4), 401-407 (1997).

12. V. V. Tuchin, Tissue Optics: Light Scattering Methods and Instruments for Medical Diagnosis, 3rd Ed., Bellingham: SPIE Press (2015).

13. V. V. Tuchin, Optical Clearing of Tissues and Blood, Bellingham, SPIE Press (2006).

14. E. A. Genina, A. N. Bashkatov, and V. V. Tuchin, "Tissue optical immersion clearing," Expert Rev. Med. Devices 7(6), 825-842 (2010).

15. K. Becker, N. Jährling, S. Saghafi, R. Weiler, and H-U. Dodt, "Chemical clearing and dehydration of GFP expressing mouse brains," PLoS One 7(3), e33916 (2012).

16. K. Becker, N. Jährling, S. Saghafi, and H-U. Dodt, "Dehydration and clearing of whole mouse brains and dissected hippocampi for ultramicroscopy," Cold Spring Harbour Protocol 2013(7), 683-684 (2011).

17. D. Zhu, K. V. Larin, Q. Luo, and V. V. Tuchin, "Recent progress in tissue optical clearing," Laser Photonics Rev. 7(5), 732-757 (2013).

18. L. M. Oliveira, M. I. Carvalho, E. M. Nogueira, and V. V. Tuchin, "Diffusion characteristics of ethylene glycol in skeletal muscle," J. Biomed. Opt. 20(5), 051019 (2015).

19. V. V. Larin, M. G. Ghosn, A. N. Bashkatov, E. A. Genina, N. A. Trunina, and V. V. Tuchin, "Optical clearing for OCT image enhancement and in-depth monitoring of molecular diffusion,” IEEE J. Select. Tops. Quant. Electr. 18(3), 1244-1259 (2012).

20. J. Wang, Y. Zhang, P. Li, Q. Luo, and D. Zhu, "Review: Tissue optical clearing window for blood flow monitoring," IEEE J. Select. Tops. Quant. Electr. 20(2), 92-103 (2014).

21. O. Nadiarnykh, and P. J. Campagnola, "SHG and optical clearing," in Second Harmonic Generation Imaging, F. S. Pavone, and P. J. Campagnola (eds.), CRC Press, Taylor \& Francis Group, Boca Raton, London, NY, 169-189 (2014).

22. E. A. Genina, A. N. Bashkatov, and V. V. Tuchin, "Glucose-induced optical clearing effects in tissues and blood," Chap. 21 in Handbook of Optical Sensing of Glucose in Biological Fluids and Tissues, V. V. Tuchin (ed.), Taylor \& Francis Group LLC, CRC Press, London (2009).

23. A. Kotyk, and K. Janacek, Membrane Transport: An interdisciplinary Approach, Plenum Press, New York (1977). ISBN: 978-1-4684-3333-3.

24. H. Suhaimi, S. Wang. T. Thornton, and D. B. Das, "On glucose diffusivity of tissue engineering membranes and scaffolds," Chemical Engineering Science 126, 244-256 (2015).

25. J. M. Christensen, M. C. Chuong, H. Le, L. Pham, and E. Bendas, "Hydrocortisone diffusion through synthetic membrane, mouse skin and epiderm ${ }^{\mathrm{TM}}$ cultured skin," Archives of Drug Information 4(1), 10-21 (2011).

26. P. N. Skandamis, and G.-J. E. Nychas, "Preservation of fresh meat with active and modified atmosphere packaging conditions," Int. J. of Food Microbiology 79(1-2), 35-45 (2002). 
27. G. H. Zhou, X. L. Xu, and Y. Liu, "Preservation technologies for fresh meat - A review," Meat Science 86(1), 119-128 (2010).

28. D. Tiroutchelvame, V. Sivakumar, and J. P. Maran, “Optimization of mass transfer parameters during osmotic dehydration of momordica charantia slices," J. of Food Processing and Preservation, (2015).

29. A. Bouchoux, H. Roux-de Balmann, and F. Lutin, "Nanofiltration of glucose and sodium lactate solutions: Variations of retention between single- and mixed-solute solutions," J. of Membrane Science 258(1-2), 123132 (2005).

\section{Introduction and theoretical background}

The treatment of biological tissues and blood with chemical compounds is widely used in many applications of dermatology [1], clinical research [2], and tissue or organ preservation [3]. The efficiency of the treatment can be evaluated and quantified if the diffusion properties of the chemical used are known for the desired tissue. Some results have been reported for the diffusion time and diffusion coefficients of some chemical agents in specific biological tissues but the techniques used to evaluate these parameters are diverse [4-9].

The technique of optical immersion clearing is one method where the evaluation of the diffusion properties of the optical clearing agents in tissues is of most importance. By estimating those parameters, it is possible to evaluate the necessary time to turn the tissue more transparent and to quantify that transparency as a function of the agent concentration used to treat the tissue [10]. The optical immersion clearing technique is a method initially described in 1997 [11] and presents great potential to be applied in clinical applications that use optical diagnosis or treatment methods [2]. Such technique originates a decrease in the natural scattering coefficient and an increase of the natural scattering anisotropy factor of the tissue, creating a temporary transparency that can be later reversed [12-13]. Such temporary tissue transparency is obtained by a stimulated tissue dehydration and consequent replacement of the water in the interstitial space by a biocompatible agent that has a higher RI, better matched to the RI of the other tissue components [14-18]. Recent research work demonstrated that this technique can be used in vivo with imaging techniques such as optical coherence tomography [19], speckle methods to monitor blood flow in dermis or cortical tissues [20], and second harmonic generation imaging ( $\mathrm{SHG}$ ) to improve tissue depth and resolution [21]. These various studies have also demonstrated the reversibility of the treatments applied by assisted rehydration.

The mechanisms involved in this technique are designated tissue dehydration and refractive index (RI) matching that ultimately turn the tissue more organized internally, leading to a smaller light scattering [13]. Such treatment is completely reversible by natural rehydration in vivo and by assisted rehydration on ex vivo tissue samples [10]. The potential of the optical immersion clearing technique is very significant to be used with clinical procedures that apply light for diagnostic and treatment purposes $[2,7,14]$.
Considering an ex vivo sample, as it is immersed in an aqueous solution containing a particular concentration of a biocompatible chemical agent, the agent in the solution creates an osmotic pressure over the sample, which induces water loss by the tissue. This is the dehydration mechanism, which initiates immediately as the treatment is applied to the tissue sample. This mechanism is in general fast, usually contained within the first two minutes of treatment [18]. At the same time, but with a slower pace, the agent in the solution begins to diffuse into the outer layers of the tissue [18]. The difference in viscosity properties and molecular size between water molecules and agent molecules provide that the diffusion of agent into deeper layers of tissue to create the RI matching mechanism takes a longer time [10].

Since these two mechanisms occur simultaneously at the beginning of the treatment, it is not easy to discriminate one from the other and time dependence measurements made during treatment represent the mixed global flux, which is a combination of the water flux out of the tissue and the OCA flux into the tissue. This way, the individualization of the two mechanisms needs a refined method that produces accurate results. Such a method is described in literature $[8,13]$ and we have recently proven its concept experimentally as described in our recent publications $[7,10,18]$. In those papers we describe how we have used thickness and collimated transmittance $\left(T_{c}\right)$ measurements made from thin muscle samples under treatment with glucose and ethylene glycol (EG) solutions to estimate the diffusion time, diffusion coefficient and viscosity of these agents in muscle. Our research has produced additional results. Those additional results were the estimated diffusion properties of water inside the muscle $[10,18]$ and the amount of free water content in the natural muscle [7, $10]$.

Considering as an example a treatment of a slabform tissue sample with a solution of a particular optical clearing agent (OCA), we can assume that if the solution has a significant higher volume than the sample (e.g. 10x), the OCA diffuses through both slab surfaces according to free diffusion. If the tissue sample has a thickness d, we can calculate the OCA concentration inside the tissue as a function of time as $[8,13,18]$ :

$$
C(t)=\frac{1}{d} \int_{0}^{d} C(x, t) d x \simeq C_{0}\left[1-\exp \left(-\frac{t}{\tau}\right)\right] .
$$

Eq. (1) is a first-order approximation of solution of the second Fick's law of free diffusion for an axial 
direction crossing the tissue sample [9, 11, 13, 22 - 23]. In this equation, if the OCA concentration in the immersing solution $C_{0}$ is known, the OCA concentration on the tissue can be calculated for any particular time $t$ as an exponential increase over time. Such exponential increase is characterized by the diffusion time $\tau$. If we are performing $T_{c}$ measurements during the treatment, we can correlate the $T_{c}$ time dependence with the time dependence of OCA concentration in the tissue. This way, we can rearrange Eq. (1) to fit the $T_{c}$ time dependence as follows [18]:

$$
T_{c}(\lambda, t) \propto \frac{C(t)}{C_{0}}=1-\exp \left(-\frac{t}{\tau}\right)
$$

In Eq. (2) we have indicated the wavelength $\lambda$ to say that the equation is valid for a single wavelength, but similar calculations can be performed for different wavelengths. Such equation is only valid when an effective flux is active between the immersing solution and the tissue sample. It is common in optical clearing treatments that if we perform the treatment for a long period of time, we will certainly observe a saturation regime where no active flux occurs. The time when the saturation regime begins depends on the sample, its geometry and also on the OCA concentration used to perform the treatment. As an example, for treatments of muscle samples with glucose solutions, we have observed that the beginning of the saturation regime occurs at a later period of time if we increase the glucose concentration in the solution from $20 \%$ to $40 \%$ [7]. This variation is imposed by the free water content in the tissue and the OCA concentration we use. Considering that the active water and OCA fluxes occur before the beginning of the saturation regime, to process the $T_{c}$ time dependencies, we must consider only the data between the beginning of the treatment and the beginning of the saturation regime. This delimited data is then displaced and normalized to obtain time dependencies that vary between zero and unity. This organized data is fitted with a line described by Eq. (2) and during the fitting procedure, we obtain the characteristic diffusion time, $\tau$ for the effective flux in the considered treatment. If we have similar time dependencies for other wavelengths we can perform this fitting procedure for each of the wavelengths and then calculate the mean diffusion time value for that particular treatment. Repeating such method for other treatments made with other OCA concentrations we can represent the mean diffusion time as a function of OCA concentration in the treatment solution. As we have observed from our results for the studies of muscle under treatment with glucose and EG solutions [7, 18], such graph presents the highest diffusion time for a particular OCA concentration. In the case of the muscle, such OCA concentration was $40.5 \%$ after we fitted the data points with a spline to evaluate dependence on concentration. Such highest value indicates an optimized OCA diffusion into the tissue, meaning that no water flux occurs in this particular treatment due to the balance between the tissue free water and the water in the immersing solution [18]. If the OCA concentrations in the solutions used to perform the treatments are well selected, the analysis of this final graph provides information about the free water content in the tissue under study. For skeletal muscle, we have obtained a free water content of 0.595 (1 - 0.405), which was a value previously unknown. At this OCA concentration, we have estimated the diffusion time values of glucose and EG in muscle as $302.9 \mathrm{~s}$ and $446 \mathrm{~s}$, respectively. These values characterize the RI matching mechanism of optical clearing with glucose and $\mathrm{EG}[7,18]$.

A similar procedure can be performed to estimate the water diffusion time, if we consider a treatment with a highly concentrated solution. For a treatment with $54 \%$-glucose we have obtained a diffusion time for water of $58.4 \mathrm{~s}$ and for the treatment with $60 \%$-EG we have obtained a diffusion time of $57.9 \mathrm{~s}$. Although these values are a little different, they indicate that the dehydration mechanism occurs in the muscle within the first minute of treatment.

Once the true diffusion time is obtained for OCA and water, we can use Eq. (3) to calculate the diffusion coefficient $[11,13,22-23]$ :

$$
D_{a}=\frac{d^{2}}{\pi^{2} \tau}
$$

Since the OCA flux in and water flux out of the tissue sample occur through both sample surfaces, Eq. (3) is appropriate to calculate the diffusion coefficients for these fluids [13]. Using Eq. (3) we have calculated the diffusion coefficients for glucose $\left(5.9 \times 10^{-11} \mathrm{~m}^{2} / \mathrm{s}\right)$, EG $\left(4.6 \times 10^{-11} \mathrm{~m}^{2} / \mathrm{s}\right)$ and water $\left(3.21 \times 10^{-10} \mathrm{~m}^{2} / \mathrm{s}\right.$ from the $54 \%$-glucose treatment and $3.09 \times 10^{-10} \mathrm{~m}^{2} / \mathrm{s}$ from the $60 \%$-EG treatment) [18]. Although we have obtained two diffusion coefficient values for water, they are very similar.

Finally, the dynamic viscosity of these OCAs in muscle can be calculated using the diffusion coefficients in Stokes-Einstein Eq. [24]:

$$
\eta_{O C A}=\frac{k_{B} T}{6 \pi D_{O C A} r_{O C A}}
$$

In Eq. (4), the viscosity of the OCA is calculated using the Boltzmann's constant $\left(k_{B}=1.3807 \times 10^{-23} \mathrm{~J} / K\right)$, the temperature $T$ of the sample during treatment (in Kelvin), the OCA diffusion coefficient $D_{O C A}$ and the OCA's Stoke's radius $r_{O C A}$, which should be known for the OCA in study. Performing the calculations with Eq. (4) for glucose and EG, we have obtained the values of $1.0 \times 10^{-2} \mathrm{~kg} /(\mathrm{m} \cdot \mathrm{s})$ and $1.4 \times 10^{-2} \mathrm{~kg} /(\mathrm{m} \cdot \mathrm{s})$, respectively [10]. These values indicate that muscle cell membrane limits OCA diffusion into the muscle tissue.

The diffusion of glucose and EG in skeletal muscle is one of many cases of interest and now that the theoretical methodology has been proven experimentally, similar studies need to be performed with other tissues under treatment with different 
chemicals. The collection of such information is strongly necessary for different medical and industrial related specialities. In particular the knowledge of the diffusion properties of creams and ointments in skin is very significant for cosmetics and dermatology [25]. On the other hand, the diffusion properties of low temperature preservatives are of great interest for organ preservation industry and also for food preservation in long time periods [3, 26-28]. In the particular case of tissue optical clearing, there are many tissues and many OCAs that need to be studied. A particular interesting application that can be developed is based on the differentiation between the diffusion properties of a particular OCA in healthy and pathological tissue. If this method provides significant results it will be possible to develop an optical and non-invasive diagnostic methodology for tumour detection.

With such many fields that need the collection of the diffusion and viscosity properties of chemicals in biological tissues, it is highly recommended that a software application should be developed to perform the necessary calculations from the experimental measurements to obtain the diffusion time, diffusion coefficient and viscosity of chemicals and water. We have recently developed such application as an integrated solution that performs all necessary calculations in a sequential and optimized manner to estimate the desired parameters. The application was validated with the experimental data that we have published for muscle treatments with glucose solutions and results are in good agreement [7]. The following sections describe the development and validation of the application.

\section{Materials and methods}

Since our objective was to develop a software application, we have selected MATLAB ${ }^{\mathrm{TM}}$ as our tool and programming language. The choice of MATLAB was made since it is a dedicated software to work with mathematical and physical problems and offers many possibilities in data fitting and figure generating. The main application and all subroutines were developed in MATLAB language. The final application contains many routines that are used to perform the various steps, such as load and save experimental or calculated data. Each of the steps in the data processing or calculation procedures is also made by a particular routine. The sequential steps to be executed by the application are made in an ordered manner and they will be described sequentially in section 3 .

During the application development, the experimental data that we have obtained from the muscle treatments with glucose solutions was used to make corrections to the various routines. After finalizing the application, the same experimental data was used to perform the calculations of the diffusion and viscosity properties of glucose and water. Comparing those results with the ones that we have previously obtained [10], we have validated the application.

\section{Results and discussion}

At the end of each experimental study, the only data available is the $T_{c}$ spectra and thickness time dependence measured along the various treatments with different OCA concentration [7, 10, 18]. To follow the data processing and calculation procedures described in section 1 , a routine was created to comply with each step and a main application program was developed to integrate all routines in sequential form. The main program is associated with a main window that is used to provide a user interface, present the various results in sequence to the user and allow him to adjust certain procedures manually, when necessary.

When the main program is initiated by the user, the application opens the main window. This window contains a menu at the top that allows loading or saving the experimental and calculated data, an "Options" button that allows configuring the aspect of the main window and a "Help" button that shows some guidelines to assist the user in the various stages of data processing or calculation.

To calculate data from a study, the first step is to upload the $T_{c}$ data from all treatments performed. To do this, the user selects from the "Load" menu the option "Load (New)". A browser window is opened and the user selects the main folder that contains the various sub-folders correspondent to each particular treatment with $T_{c}$ measurements (one for each OCA concentration). From the "Load" menu there is another option to load a saved experiment. Such option can be used if the user cannot finish the calculations in the same day. The upload of all data from all treatments is made at once and the spectra are presented on the right as we can see from Fig. 1.

On the upper right of the main window, we have several sub-windows that present the entire collection of spectra measured in each particular treatment. Such graphs are configured to present spectra between 170 and $1100 \mathrm{~nm}$. Although these graphs present spectra from 200 to $1050 \mathrm{~nm}$, we have neglected spectral data below $400 \mathrm{~nm}$, since it corresponds to a strong inclusion of fluorescence. As we can see from Fig. 1, the spectra presented corresponds to the treatment with $20 \%$ glucose, as indicated by highlighted label (20) on top of the graph. The other labels on the right are accessible by clicking over them and the spectra from each treatment can be seen.

Below the window with the spectra there is a matrix containing the spectral values for each time of treatment, so the user can check a particular value if necessary. If we select a different treatment above the spectra window, the data in the matrix will change automatically.

On the upper left there is an application log window that presents the various tasks that have been executed or eventual errors that might occur. Below this window there is an input window to select the wavelength bandwidth and the number of wavelengths to calculate the $T_{c}$ time dependencies. As indicated in Fig. 1, our selection was $600 \mathrm{~nm}$ for the first wavelength to be 
considered, $800 \mathrm{~nm}$ for the last and 11 for the number of wavelengths within the selected band. These choices will generate $T_{c}$ time dependencies for wavelengths at each $20 \mathrm{~nm}$ from 600 to $800 \mathrm{~nm}$. Once these values are appropriately selected, the user clicks the "Generate"

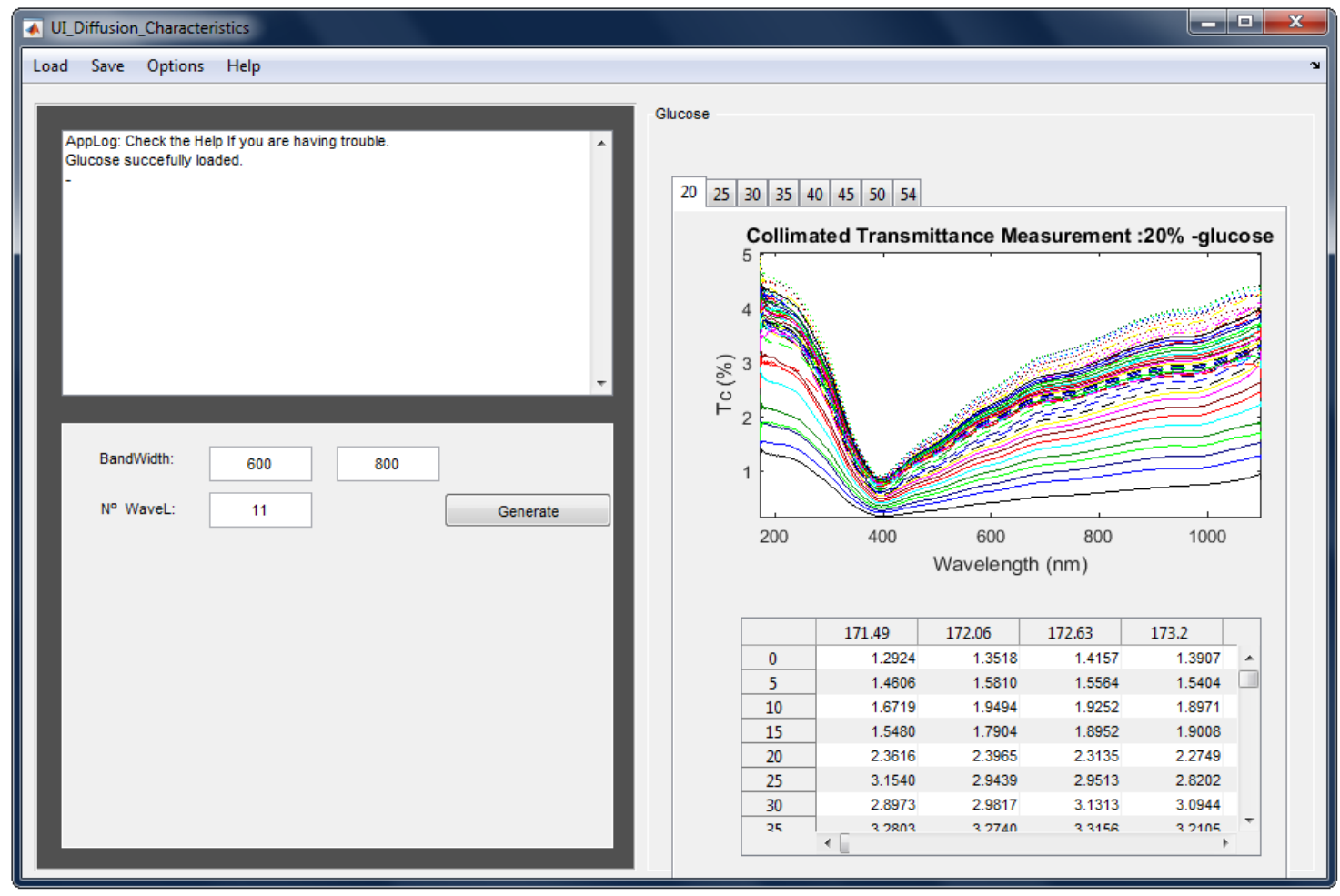

Fig. 1 Main window after uploading $T_{c}$ data from all glucose treatments.

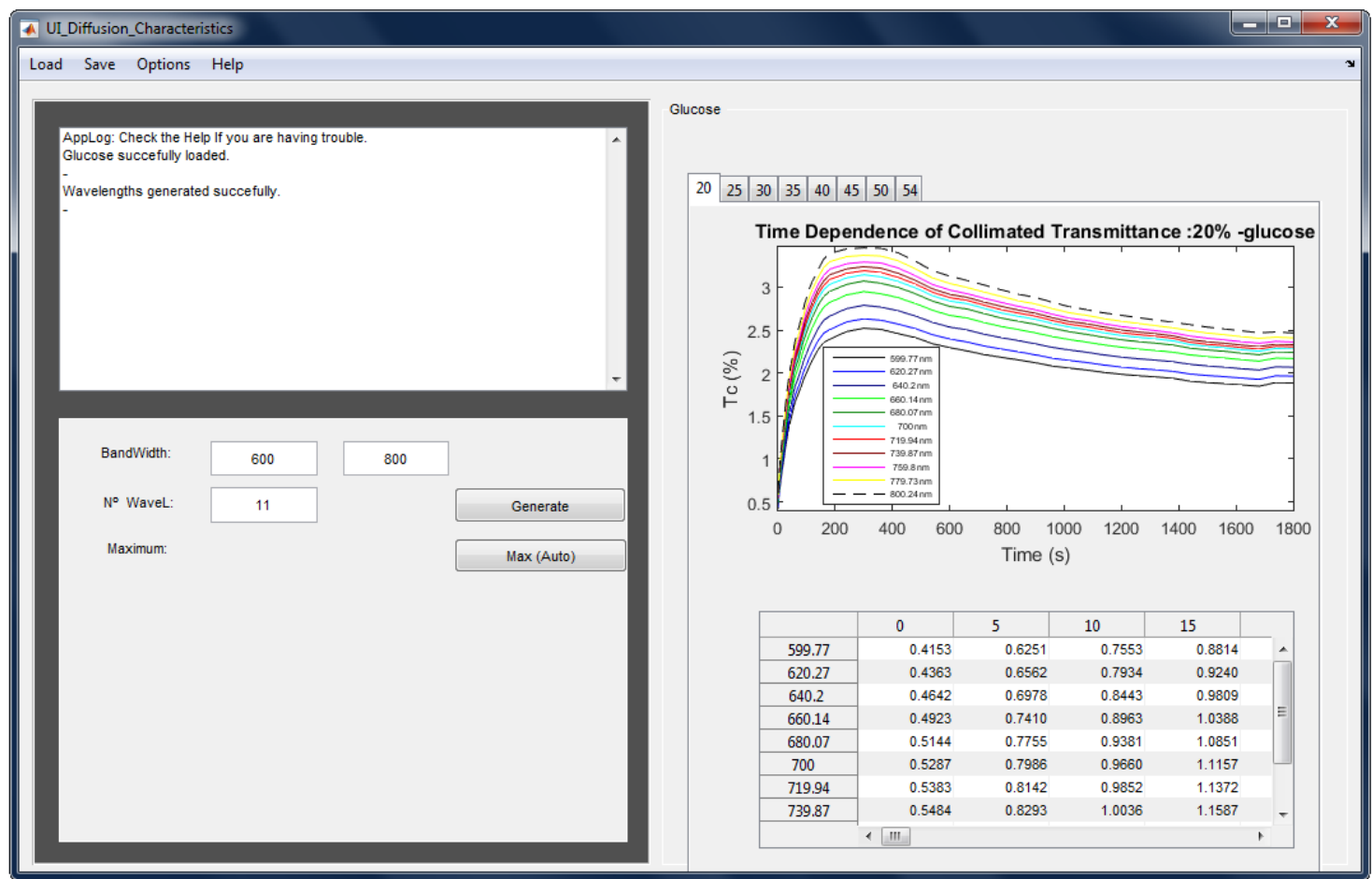

Fig. 2 Main window after selecting the wavelengths to calculate $T_{c}$ time dependencies. 
button to proceed. The previous data and graphs are replaced as can be seen in Fig. 2.

On the left of the main window a new button was added just below the "Generate" button. This new button is the "Max(Auto)" button, which allows selecting the beginning of the saturation regime in the $T_{c}$ time dependencies represented for each treatment on the upper right graphs. Once again the graphs represented on the upper right can be exchanged between treatments by selecting the desired label.
The data matrix presented on the lower right contains now the $T_{c}$ time dependencies for each wavelength and corresponds to the graph presented just above. If the graph is changed, the data in the matrix also changes. The next step consists on finding the beginning of the saturation regime for the treatment. To do this, the user selects the "Max(Auto)" button and some points appear in the graph on the right as can be seen in Fig. 3.

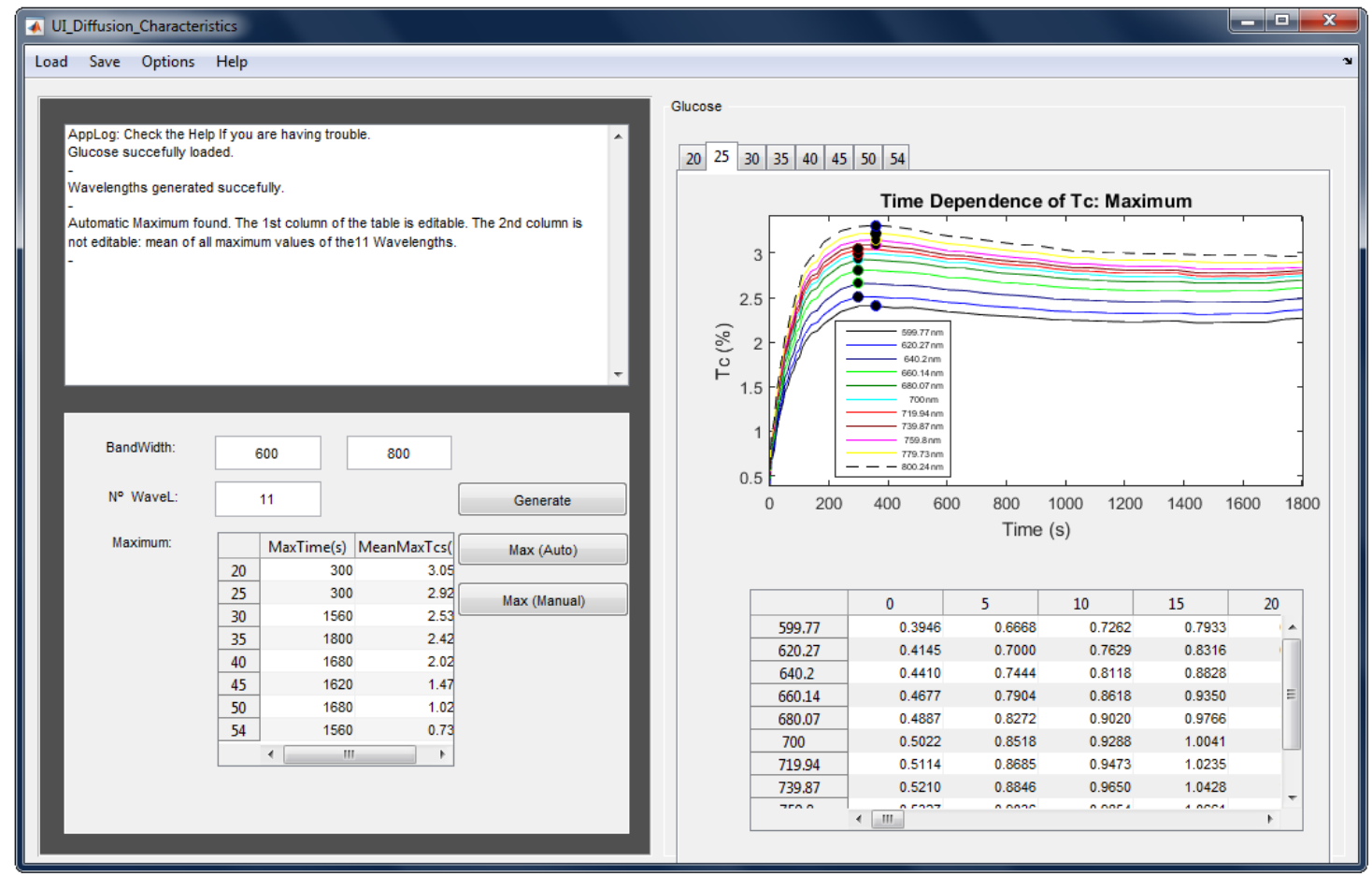

Fig. 3 Main window after automatic identification of the beginning of the saturation regime.

The graph presented in Fig. 3 corresponds to the treatment with $25 \%$-glucose to show that the automatic selection was not done accurately for this particular case. The dots presented on the various curves of the graph are seen at two different times of treatment: 300 and $360 \mathrm{~s}$ in the case presented in Fig. 3. To correct this, the user has now available on the lower left window a new button ("Max(Manual)") that allows for making a manual selection of the beginning of the saturation regime. With the automatic selection a new matrix is presented in the lower left window. In the first column of this matrix is presented for each treatment the time that corresponds to the greater number of maximal $T_{c}$ values. The second column of the matrix contains the mean maximum $T_{c}$ value observed for all dots on the graph. To correct the selection of the beginning of saturation regime, the user must click on the "Max(Manual)" button. When the user selects this button the first column on the lower left matrix becomes editable. Then the user should insert the values that he sees adequate for each treatment and click the "Max(Manual)" button again. At this point all graphs show the point markers at the time instant that the user selected, as we can see in Fig. 4 for the previous case of treatment with $25 \%$-glucose.

Now we can see in Fig. 4 that all point markers are located at 360 s for the $25 \%$-glucose treatment. After the manual correction was made, a new button appears below the "Max(Manual)" button. This new button is called "Draw [0-max]" and it shows the treatment graphs on the right delimited between the beginning of treatment and the beginning of the saturation regime. When these new graphs are generated, each dataset (for each wavelength) was displaced vertically to have $T_{c}=0$ at $t=0$. Figure 5 presents the case of $20 \%$-glucose treatment with graph contained in the first $300 \mathrm{~s}$. 


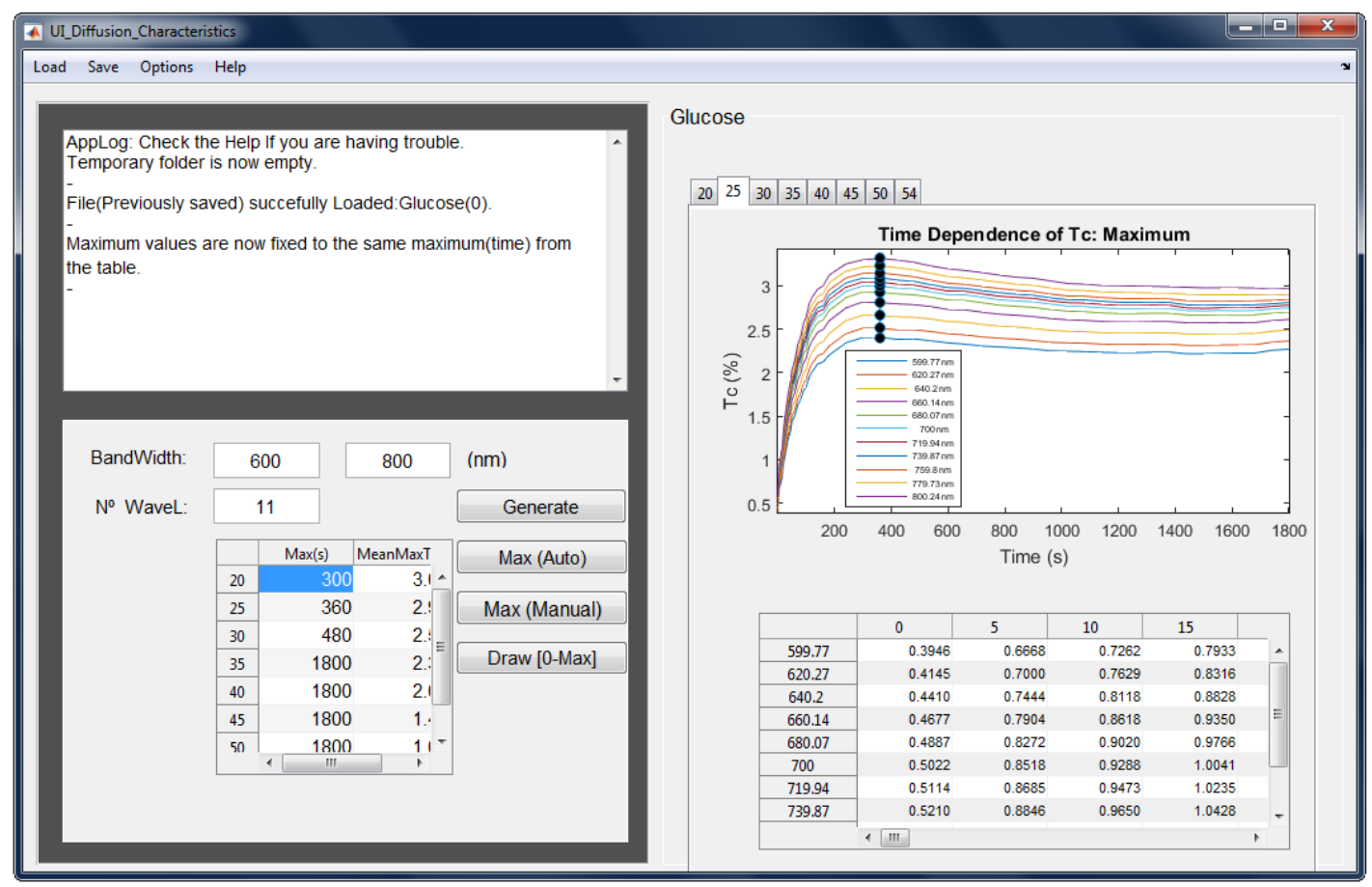

Fig. 4 Main window after manual correction of the beginning of the saturation regime.

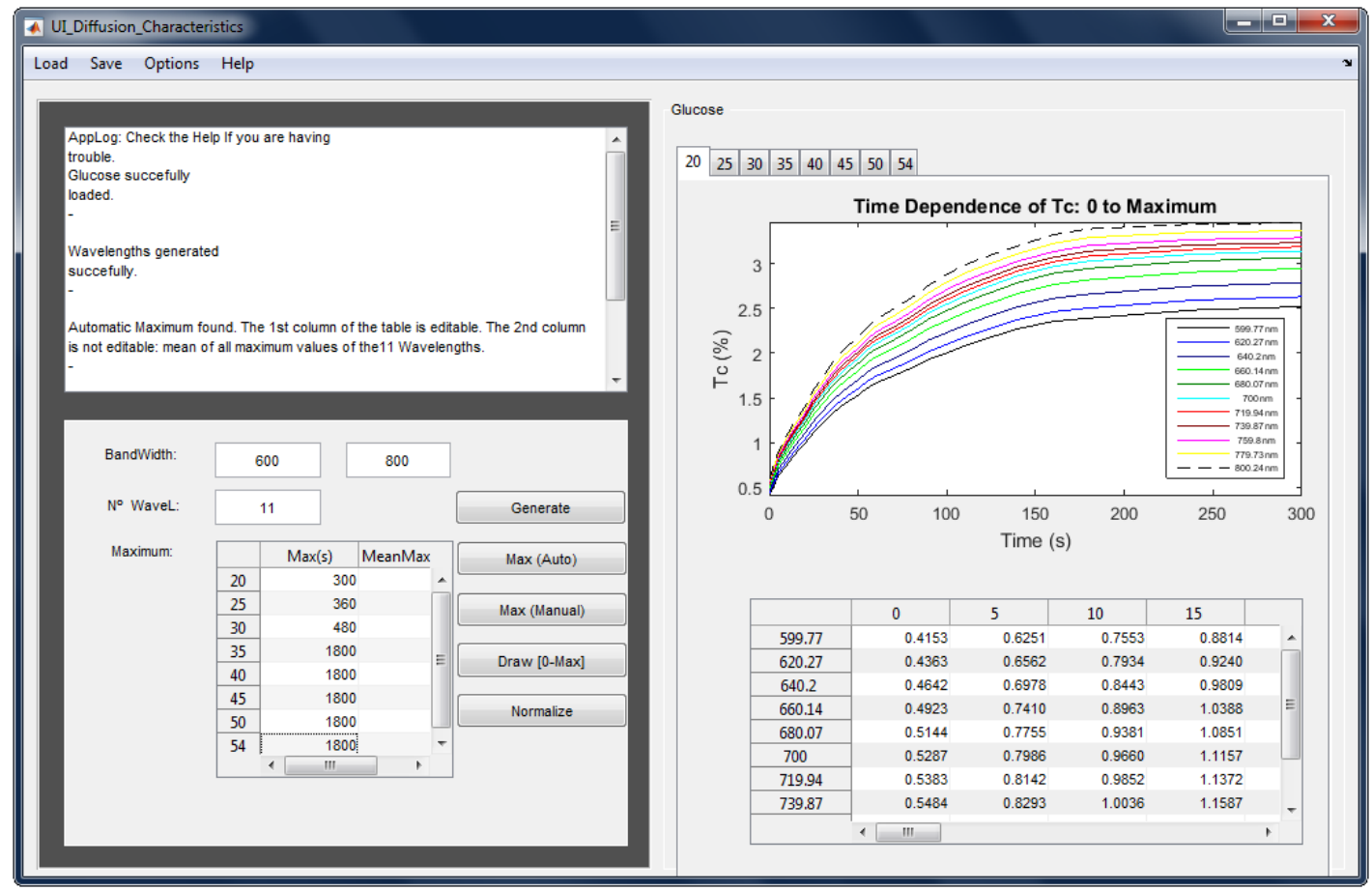

Fig. 5 Main window after $T_{c}$ time dependency delimited by the beginning of the saturation regime was displaced vertically.

Once again the matrix on the lower right of the main window contains the data values correspondent to the graph above and it is updated each time the user selects one of the treatments from the labels above graph. In Fig. 5 we can now see a new button called "Normalize". This button performs the next data processing, by normalizing the delimited $T_{c}$ time dependencies of each treatment to each highest value. This normalization procedure takes some time to be done, since normalization is made for each curve that corresponds to a single wavelength within each of the various treatments. At the end new graphs are presented on the right of the main window with the datasets normalized. 


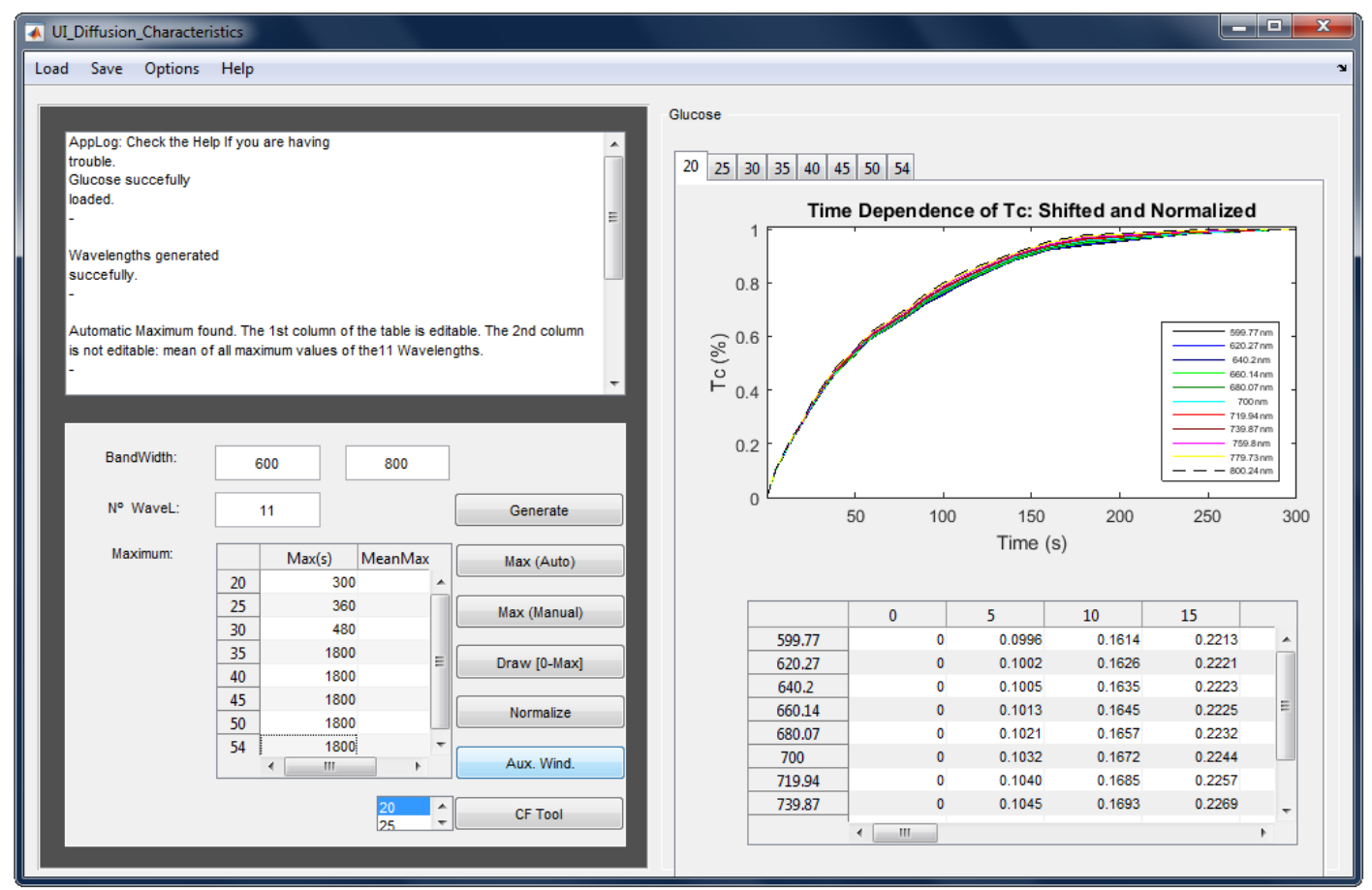

Fig. 6 Main window after performing data normalization to the value measured at the beginning of the saturation regime.

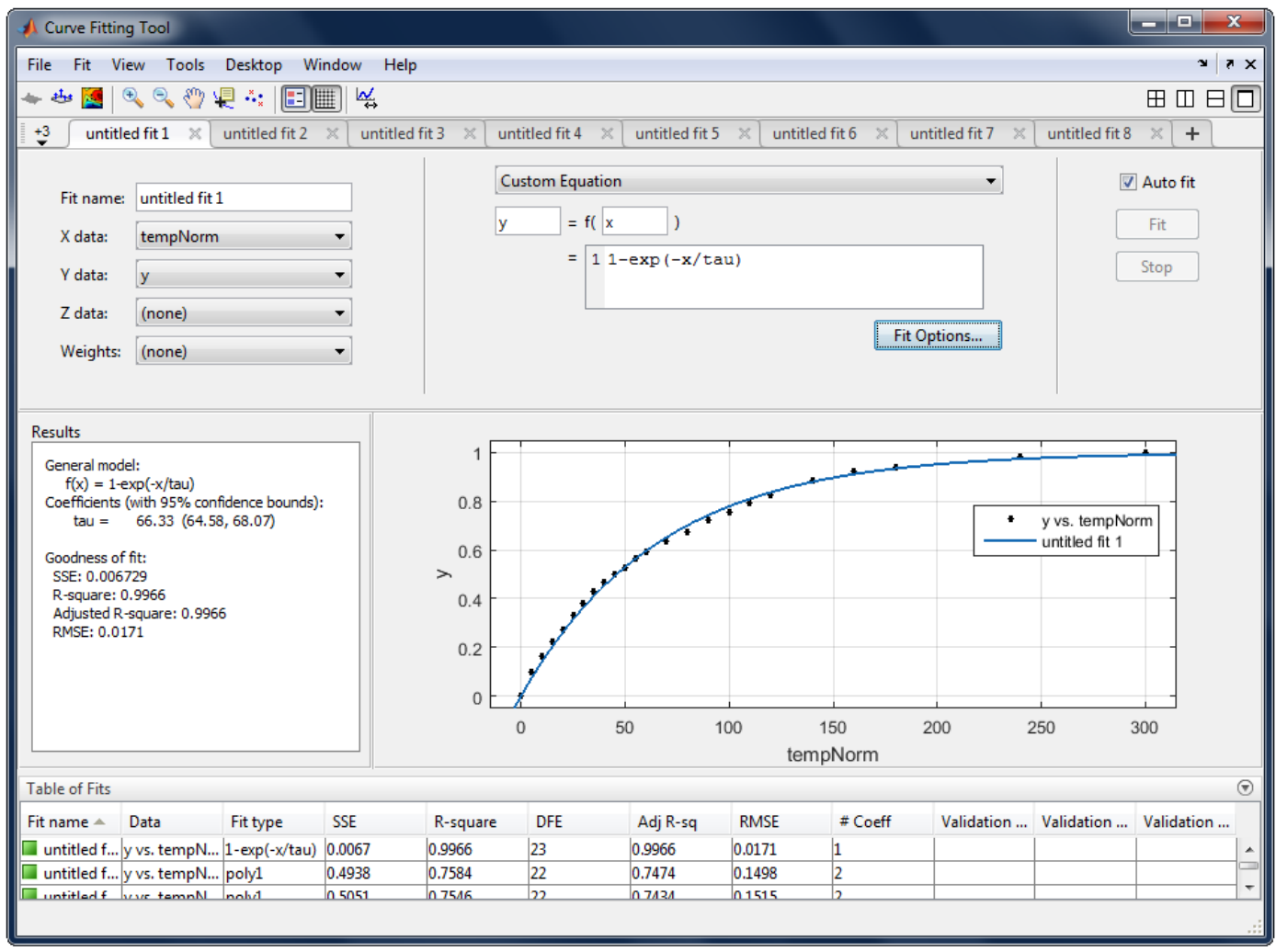

Fig. 7 Curve Fitting Tool window with all the data for the treatment with $20 \%$-glucose. 
After performing data normalization, two new buttons appear at the lower left section of the main window. These buttons will be used for the data fitting. The first new button is the "Aux. Wind.", which generates a new auxiliary window for the fittings and the second button is the "CF Tool", which corresponds to a MATLAB tool - Curve Fitting Tool and it generates also a new window with all the datasets for a particular treatment. In Fig. 6 we see that next to the $\mathrm{CF}$ Tool there is a scroll-dawn window with the number 20 in blue. This means that if the user clicks the "CF Tool" button, the data that will be uploaded to the new $\mathrm{CF}$ Tool window for the fitting procedure will be the data for the treatment with $20 \%$-glucose. If the user wishes to select another treatment, it just needs to select it from the scroll-dawn window. Considering the $20 \%$-glucose treatment, Fig. 7 presents the CF Tool window.

As we can see from Fig. 7 there are many tabs at the top with the labels "untitled fit 1", "untitled fit 2 " and so on. Each of these tabs contains the dataset correspondent to each wavelength so the user can make the data fit for each one independently. In Fig. 7 the first tab is selected with the fitting line already applied to the dataset correspondent to $600 \mathrm{~nm}$. Since CF Tool is a specific tool of MATLAB, we could not find a way so far to name the labels according to the wavelength, but this is considered for next improvements.
There are many fitting options in the window. For instance, above the graph we have the equation fitting options where the user can select the most appropriate from a wide set of equations. In our case, the equation to be used in the fitting is according to Eq. (2). By selecting a custom equation and introducing Eq. (2) manually, the user can then select the best fitting options for this type of line by clicking the "Fit Options..." button. The fitting is optimized when the Rsquare value on the "Results" window is maximized. In the case represented in Fig. 7, the R-square value is 0.9699 and the $\tau$ value obtained in this fitting is $66.33 \mathrm{~s}$.

The R-square value optimization is made through a set of options related to fitting methods. Within the Leastsquare fitting method, we can for instance select between the algorithms of "Trust-Region" or "Levenberg-Marquardt". The objective is to obtain the highest R-square value to ensure the best data fit. A fitting must be performed to all datasets correspondent to each of the 11 wavelengths (one in each tab). The user must do these fittings manually and sequentially. Once all fittings are made and optimized, the correspondent $\tau$ values can be introduced manually in the matrix of the auxiliary window:

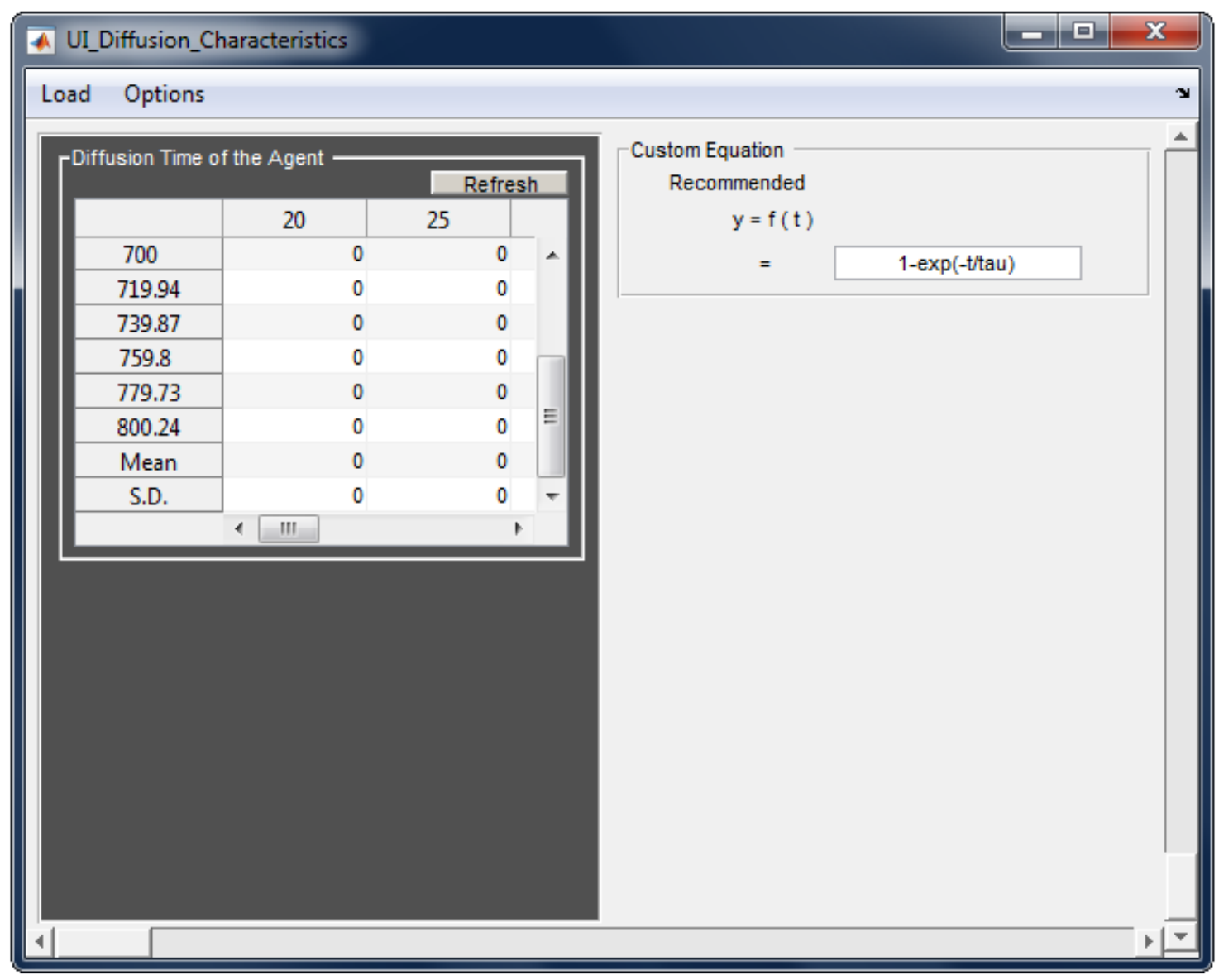

Fig. 8 Auxiliary window to introduce the diffusion time values for each treatment. 
The auxiliary window represented in Fig. 8 contains the fitting equation on the right side, so that the user can copy it to the CF Tool window to save time. Both windows must be open at the same time to copy data manually from one to the other. The user must introduce the values on the matrix of the auxiliary window manually, since there is no direct way to copy those values from the "CF Tool" window.

Once the matrix in the auxiliary window is complete with all the diffusion time values from all wavelengths and treatments, the user clicks on the "Refresh" button above the matrix and the mean and standard deviation for all treatments are calculated and presented in the two lower rows of the table. At the same time a graph appears at the lower right of the auxiliary window containing the mean diffusion time values as a function of OCA concentration in solution. This graph shows also error bars that correspond to the standard deviation values on the matrix to show how disperse are the diffusion times in each treatment.

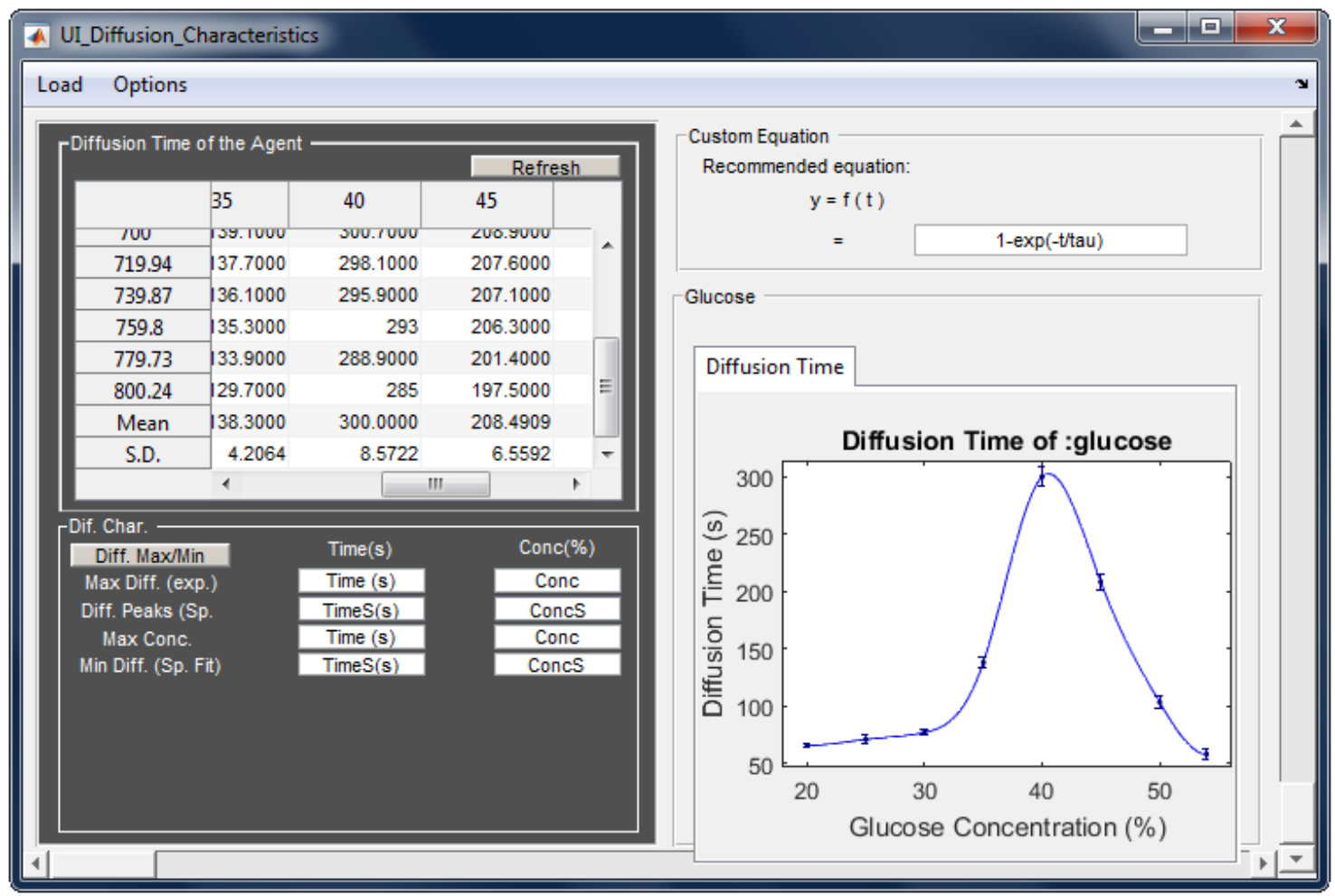

Fig. 9 Auxiliary window after filling the $\tau$ matrix.

As we can see from Fig. 9, some fields related to the OCA and water diffusion characteristics also appeared at the lower left. The first column corresponds to the diffusion time and the second to the OCA concentration in solution where those diffusion time values are observed. Once the user clicks on the "Diff. Max/Min." button, the values in these fields are automatically filled and new fields appear just below, as presented in Fig. 10.

In the first column of the diffusion characteristics, we see from top to bottom: the maximum experimental mean diffusion time (300s), the estimated absolute maximum mean diffusion time $(302.674 \mathrm{~s})$, the smallest experimental mean diffusion time $(58.4 \mathrm{~s})$ and the smallest estimated mean diffusion time (58.4s). The second column contains the OCA concentration values that correspond to the diffusion time values in the first column. As an example, the estimated maximum diffusion time of $302.674 \mathrm{~s}$ would occur for an OCA concentration of $40.6 \%$. The values in the two columns are also presented on the right side graph with different symbols and colours.

As the diffusion characteristic values are presented, four new fields are presented just below. These fields are the sample thickness (left column) and the diffusion time (right column) to calculate the diffusion coefficients for OCA and water. The user must copy the diffusion time values from OCA (302.674s) and water (58.4s) into the lower second column. These values are much approximated to the ones that we have previously estimated for glucose. In our previous estimations [7, 18], we have estimated a diffusion time of 302.9s for glucose from a treatment with $40.5 \%$-glucose and the exact diffusion time of $58.4 \mathrm{~s}$ for water from a treatment with $54 \%$-glucose.

Once the first of these diffusion time values is introduced, new other fields and buttons appear, as we can see from Fig. 11. 


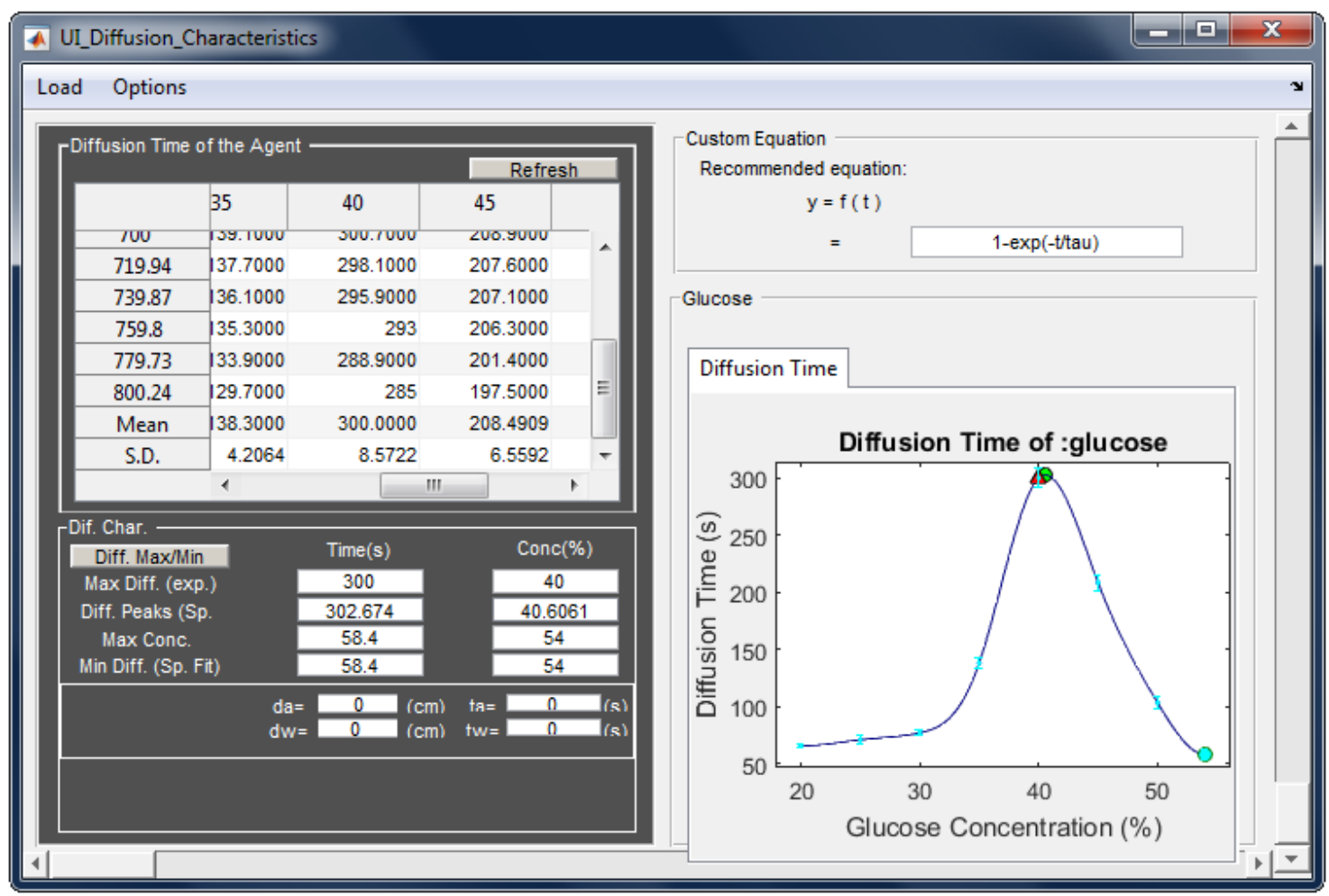

Fig. 10 Auxiliary window with automatically detected data for the diffusion time values.

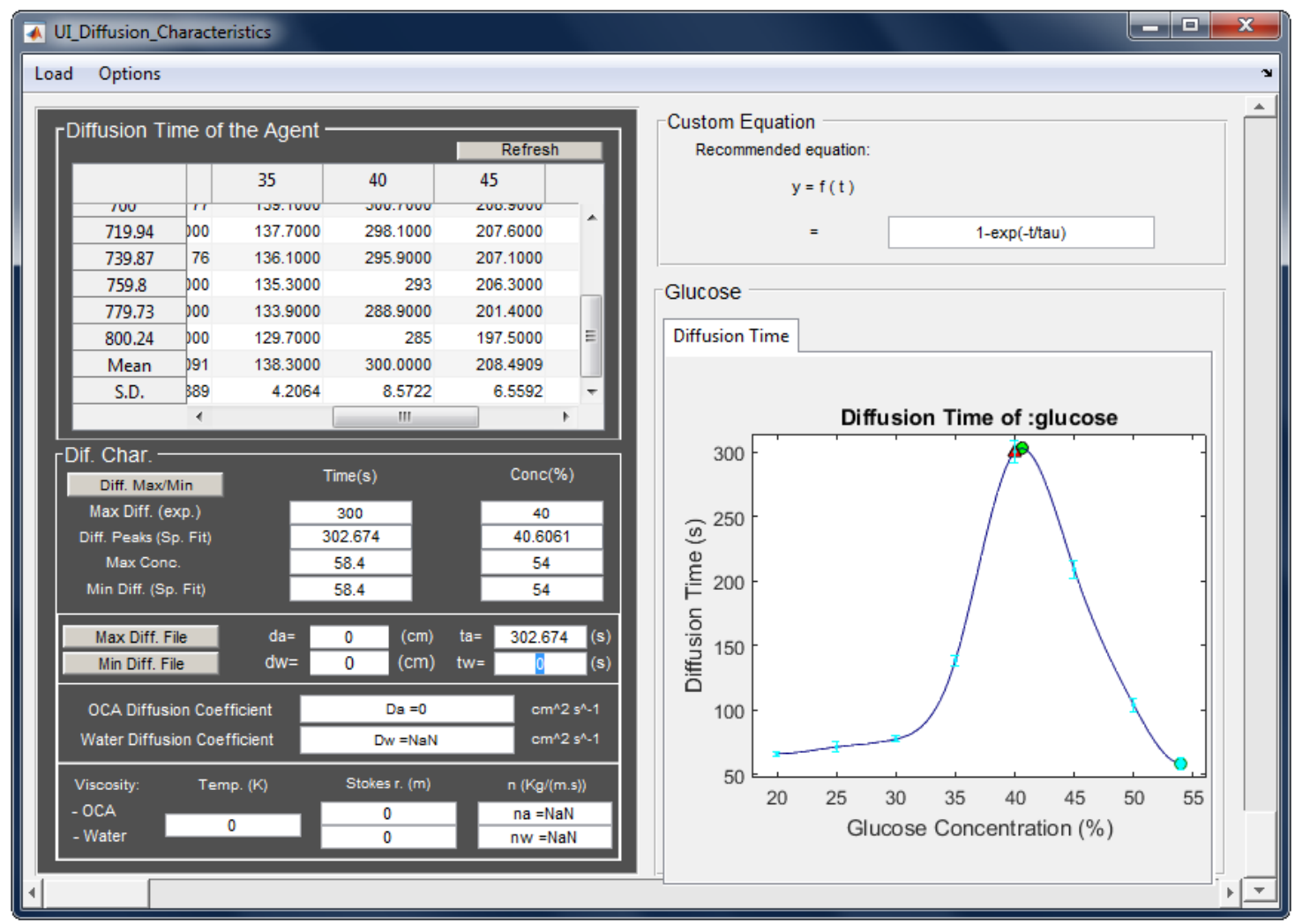

Fig. 11 Auxiliary window ready to calculate the diffusion coefficient and viscosity values.

Figure 11 shows that two new buttons became available on the left side of the thickness fields. If the user has the correct values for sample thickness to introduce, he can do it manually. If not, the new buttons are used to upload the experimental thickness time dependence files that correspond to the OCA 
concentrations that produce the diffusion coefficients for OCA and water. For the case of water diffusion time, the OCA concentration was $54 \%$, so we can upload the thickness time dependence of the sample for this treatment, by clicking the "Min Diff. File" button. For the case of OCA diffusion time, the application estimated that an OCA concentration of $40.6 \%$ is the ideal one. This value is almost the same as the ideal glucose concentration obtained in our previous estimations (40.5\%) [18]. Since we do not have data for such a treatment, we can upload the thickness time dependence for the treatment with $40 \%$-glucose by clicking the "Max. Diff File" button. By using this data, we do not introduce a significant error.
At the bottom, we already see from Fig. 11 the fields to present the diffusion coefficients and viscosity values of OCA and water. After introducing the diffusion time values for OCA and water, the user uploads the thickness time dependence files. Once they are uploaded, the application immediately creates two new graph tabs on the right. These new graphs contain the thickness time dependencies that were uploaded. The application also creates in each of the graphs a point with the thickness value that corresponds to the diffusion coefficient presented on the left. If the diffusion time value does not correspond exactly to a particular measurement, the point is interpolated, as we can see from graphs in Figs. 12 and 13.

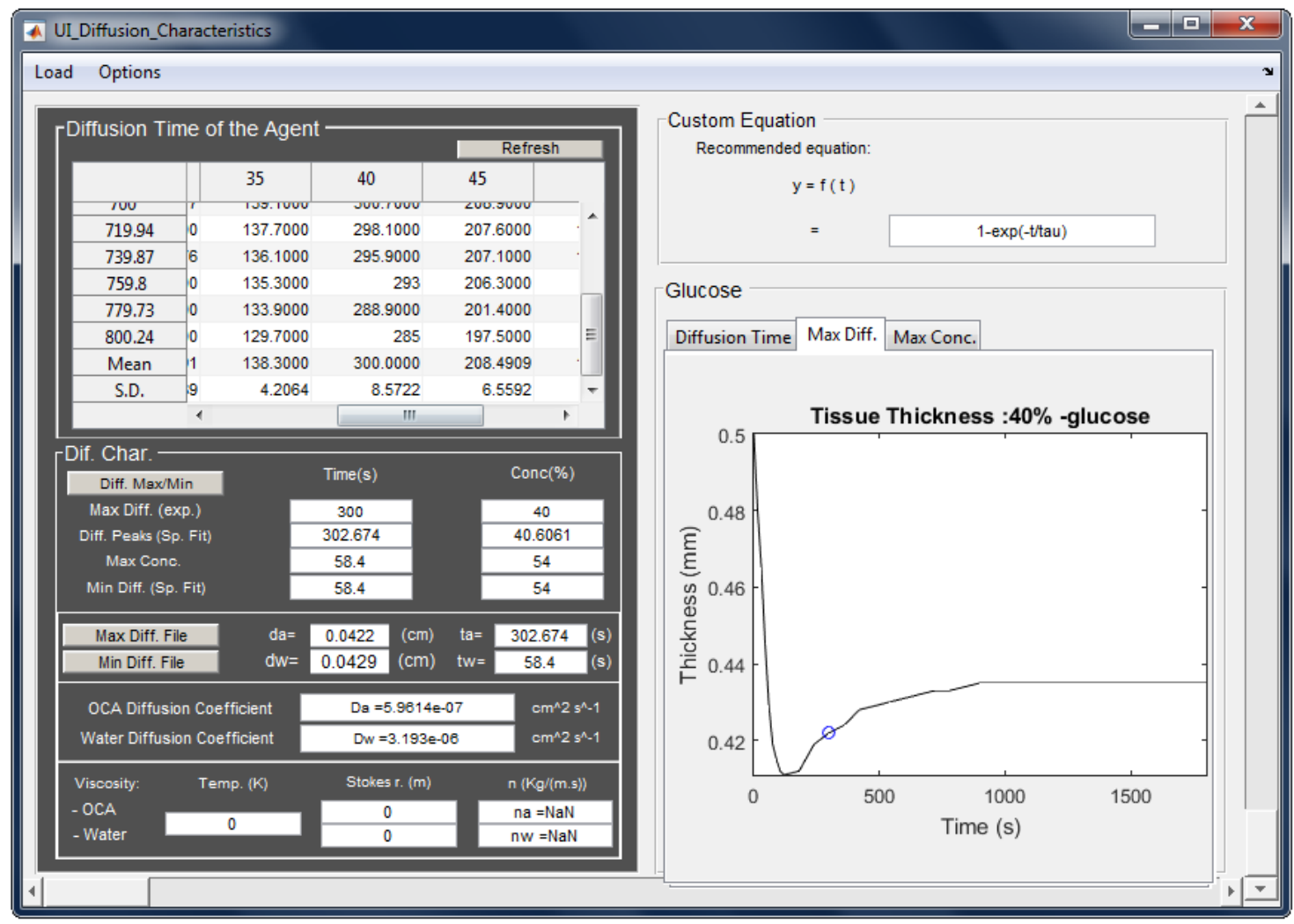

Fig. 12 Thickness time dependence graph for the treatment with $40 \%$-glucose.

For the case presented in Fig. 12, a blue dot was automatically placed at the time of $302.674 \mathrm{~s}$ and the correspondent thickness value of $0.0422 \mathrm{~cm}$ is placed in the field on the left side of the auxiliary window. The same happens to determine sample thickness to calculate water diffusion coefficient, as presented in Fig. 13.

In this case, a thickness of $0.0429 \mathrm{~cm}$ corresponds to the $58.4 \mathrm{~s}$ in the treatment with $54 \%$-glucose.

In addition to the automatic detection of sample thickness, the diffusion coefficients are also automatically calculated and presented in the corresponding fields.

As we can see from Figs. 12 and 13, the calculated diffusion coefficients for glucose and water are respectively $5.9614 \times 10^{-7}\left(\mathrm{~cm}^{2} / \mathrm{s}\right)$ and $3.193 \times$ $10^{-6}\left(\mathrm{~cm}^{2} / \mathrm{s}\right)$. For the diffusion coefficient of glucose in muscle, our previous calculations have estimated the value of $5.9 \times 10^{-7}\left(\mathrm{~cm}^{2} / \mathrm{s}\right)$ [10]. Our previous estimation for the water diffusion coefficient was $\left(3.21 \times 10^{-6}\left(\mathrm{~cm}^{2} / \mathrm{s}\right)\right)$ [10]. By comparing between these values, we see that the application generates very similar values. The differences that were observed have to do with the possibly different fitting algorithms and options used in both estimations to determine the various $\tau$ values.

The final calculation is the viscosity of glucose and water. To perform this calculation, the user must introduce the temperature (in K) observed during the studies and Stokes radius in the auxiliary window. This 
value must be in meters (m), as indicated on Fig. 13, for example. For the temperature value we have introduced $293 \mathrm{~K}\left(20^{\circ} \mathrm{C}\right)$, as in our previous estimations. Since we do not know the Stokes radius for water molecules, we have only introduced the value for glucose. This value of $0.365 \times 10^{-9} \mathrm{~m}$ is the same used in our previous estimations and was obtained from literature [29]. Fig. 14 shows the auxiliary window with all the data calculated.

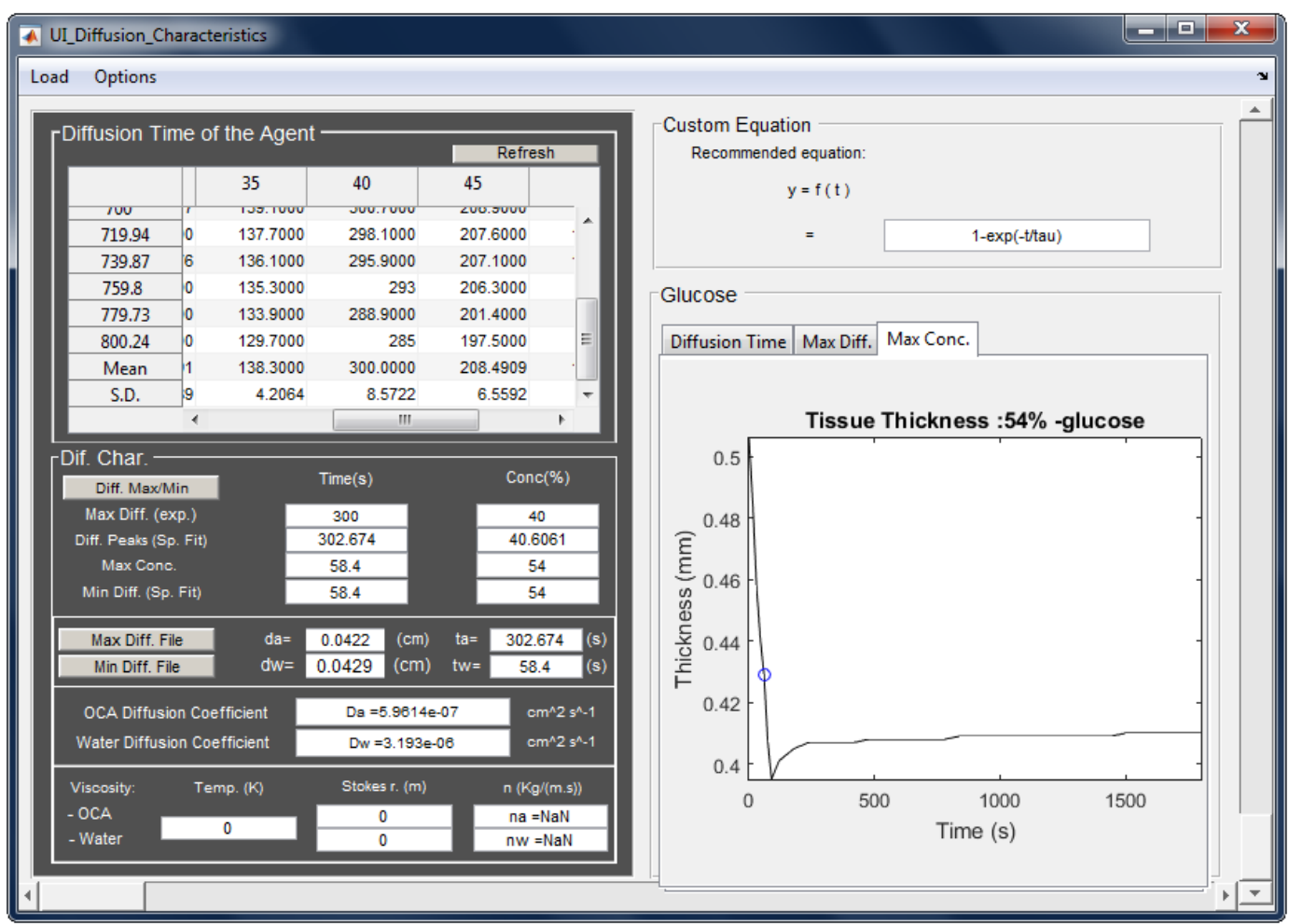

Fig. 13 Thickness time dependence graph for the treatment with 54\%-glucose. Blue dot signals the thickness at 58.4s.

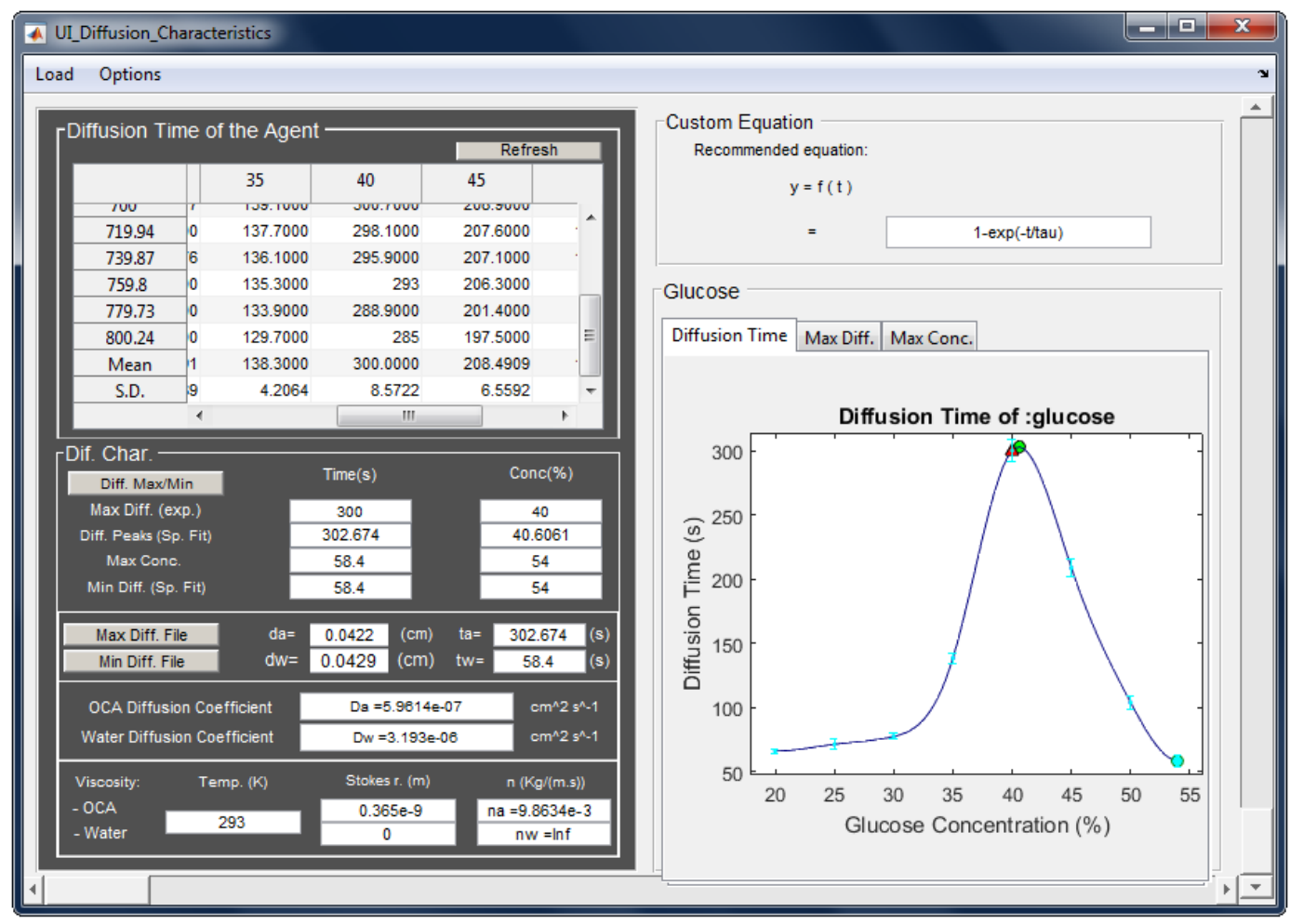

Fig. 14: Complete auxiliary window with all estimated and calculated diffusion and viscosity data. 
Comparing the calculated viscosity for glucose $\left(9.863 \times 10^{-3}(\mathrm{~kg} /(\mathrm{m} \cdot \mathrm{s}))\right)$ with the one from our previous estimations $\left(1.0 \times 10^{-2}(\mathrm{~kg} /(\mathrm{m} \cdot \mathrm{s}))\right)$ [10], we see that they are also much approximated.

Although there have been observed some limitations in the application, it works in an integrated manner to perform the necessary calculations from the raw $T_{c}$ and thickness measurements. There are some improvements that we plan to do to this application to turn it even better. We will discuss these matters in the following section.

\section{Conclusions and future perspective}

After finalizing, testing and validating the application we see that it is a very useful tool to help in the calculation of the diffusion properties of chemicals and water in biological tissues. It is an organized and focused tool that uses the theoretical background described in literature [13] for fast estimation of the characteristic diffusion and viscosity properties of optical clearing agents and water in tissues, based on collimated transmittance $T_{c}$ and thickness measurements of a tissue sample. Such properties are particular for any agent-tissue combination and allow the characterization of the optical clearing mechanisms involved in the treatment - tissue dehydration and RI matching. Once the software has been developed, it can now be used for several and diversified applications. For instance, future studies can now be performed to calculate the diffusion properties of skin lotions and topically delivered medications. Another potential application may provide a diagnosis method for cancer detection. Healthy and pathological tissues have different water contents and consequently will originate different diffusion properties or viscosities for agents used in a monitoring study. By comparing between the different calculated properties, it might be possible to develop a noninvasive optical diagnosis method for early cancer detection. Future studies are necessary and this software is a significant asset for that research.

This application can read any set of files, provided they are named in a pre-established way and placed inside folders that correspond to particular treatments. Although the case presented here corresponds to treatments with glucose at the specified concentrations in solution, other concentrations are accepted by the application. It also can export graphical and numerical data in different formats like excel, pdf or MATLAB figures. The figures were not presented in this paper, but they can be generated and formatted according to the wishes of the user. At any stage of the data processing or calculation, a report can be generated in pdf or latex formats.

There are also some things we wish to improve in this application. As mentioned above, one thing to be tried in the next improvements is to correct the graphical labels in the $\mathrm{CF}$ Tool window to indicate which wavelength corresponds to each dataset to be fitted. On the other hand, the application saves temporary files with the data from the various steps of processing or calculation. One other improvement is to provide these file savings in a more user friendly manner.

Considering the benefit of having such application, we now plan to use it in our future research. As we have indicated in the introduction, we plan to perform several and different diffusion studies of various chemicals in various animal and human tissues. We will use this application to facilitate our data processing and calculation to obtain the diffusion properties in a more easier and fast way. 